\title{
A computer-controlled four-roll mill for investigations of particle and drop dynamics in two-dimensional linear shear flows
}

\author{
By B. J. BENTLEY† AND L. G. LEAL \\ Chemical Engineering Department, California Institute of Technology, \\ Pasadena, CA 91125, USA
}

(Received 6 June 1985 and in revised form 21 December 1985)

In this paper we describe the design and operating characteristics of a computercontrolled four-roll mill for investigations of particle and drop dynamics in twodimensional linear flows. The control system is based upon the use of: a video camera to visualize the instantaneous position of the drop or particle; a PDP 11/23 computer, with a pipeline processor acting as an interface between the camera and computer, to calculate the position and implement a control strategy, and d.c. stepping motors to convert an electronic signal to angular velocities of the four rollers. The control objective is to keep the centre of mass of the drop/particle at the centre of the region between the rollers where there is a stagnation point in the undisturbed flow, while maintaining the shear-rate and the ratio of vorticity to strain rate in the flow at fixed values. The resulting system is suitable for studies of: the rotational motions of single solid particles; the deformation and burst of single droplets; or the hydrodynamic interactions of two particles or drops, one of which is held with its centre-of-mass fixed at the stagnation point of the undisturbed flow. In all cases, the flow can be varied from pure rotation to pure strain, and the shear rate can be either steady or changing as a prescribed function of time.

\section{Introduction}

There are many circumstances in which it would be of interest to investigate experimentally the dynamics of small particles or drops in a viscous fluid which is undergoing a linear flow. Specifically, in suspensions, emulsions and other multiphase fluids, the lengthscale characteristic of the dispersed or particulate phase is often small compared with that characteristic of the flow as a whole so that the motion can be approximated locally (i.e. on the particulate scale) as linear. In that context, it is basically the rotation, deformation and interactions of particles or drops that are of primary interest. A key feature of particle or droplet motion is that it is expected to depend critically upon the relative magnitudes of the vorticity and strain rates of the undisturbed motion; for example, the behaviour of viscous drops is known to be profoundly different in a hyperbolic straining flow (for which the vorticity is zero), and a simple shear flow with the same magnitude of the velocity gradient. Thus, there is a strong motivation to investigate particle and drop dynamics in a wide variety of linear flows, representing the spectrum from purely rotational flows, on the one hand, to purely extensional (or straining) flows on the other.

An experimental apparatus that appears ideally suited to studies of this type is

† Current address: Dynamic Solutions, 2355 Portola Road, Ventura, CA 93003, USA. 
the so-called 'four-roll mill', a device invented in the 1930s (Taylor 1934), for studies of drop deformation and burst in two-dimensional, pure straining flows. This device produces a very good approximation to a linear flow in the region between the four rollers, and it allows the magnitude of the rate-of-strain tensor to be arbitrarily selected relative to the vorticity (Giesekus 1962) by various combinations of roller speed and direction. Furthermore, there is a stagnation point located centrally between the rollers for any of the possible flows. Thus, in principle, the particle or drop of interest will remain at a fixed position in the device, even if the flow is time-dependent. Potentially, then, the four-roll mill could be used to study such problems as the deformation of drops or deformable particles in steady or timedependent, two-dimensional flows with an arbitrary ratio of strain rate to vorticity; the rotational motion of a rigid, non-spherical particle under the same circumstances; the deformation or breakup of 'flocs' of many particles held together by weakly attractive physico-chemical forces; or the hydrodynamic interaction (including 'capture') between two particles, two drops, or a particle and a drop by placing one at the stagnation point of the flow while the other moves through the device. Unfortunately, however, implementation of such studies has been severely restricted because the stagnation point of the flow is not a stable equilibrium position for a particle or drop; indeed, the experimental difficulty of maintaining a drop or particle at a fixed position for observation has greatly inhibited (or in some cases prevented altogether) the use of the four-roll mill for this type of investigation. The primary application has been to studies of drop deformation and burst by Taylor (1934), and subsequent investigators (Rumscheidt \& Mason 1961 ; Grace 1971). However, these studies have been restricted to pure straining motion since it is only in this case that approximate manual control of drop position is possible, and even then the achievable velocity gradients were very small and the flow resulting from the 'control' process was highly unsteady.

In this paper we describe a unique, new implementation of the four-roll mill in which automatic, computer-control of the flow device is used to maintain a particle or drop at the stagnation point for all the spectrum of possible flow types, including both steady and time-dependent flows, with minimal disruption of the flow. Our immediate goal in designing and building this device was an experimental investigation of the deformation and burst of a single viscous drop in a second, immiscible Newtonian fluid. However, the resulting apparatus is suitable, with minor modification, to all of the problems that were described above (as well, presumably, of others which we have not thought about).

Three difficulties are encountered in automating the control of a drop or particle. The first is the difficulty in accurately locating the position of the drop (which must be done non-invasively), the second is the calculation of the roller speeds required to adjust the flow field to maintain the drop near the device centre (which requires a digital computer), and the third is the implementation of these required motor speeds. We were able to solve the first problem by interfacing a digital video camera to a laboratory computer to sense the drop position. The computer was used to perform the roller-speed calculations, and was also interfaced to four independent stepping motors to effect the roller speeds thus calculated. The technology in both digital imaging and laboratory computers has only recently become economical enough to make this feedback control scheme feasible. In many ways our device resembles a robotic system in which the video camera provides the eye and the digital computer the brain. Indeed, in designing the video-camera/computer-based control system, we have utilized image-processing technology from the robotics programme at the Jet 


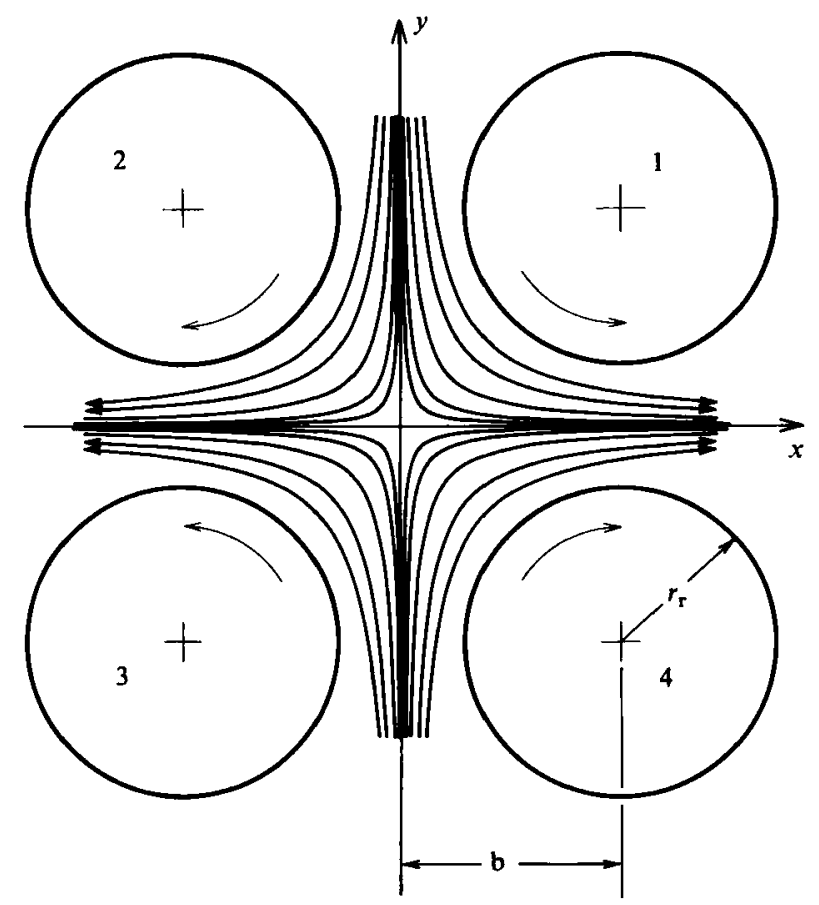

FIGURE 1. Schematic of four-roll mill (top view).

Propulsion Laboratory to develop an interface for reducing the data stream from the video camera to a size that is compatible with the use of a relatively small laboratory computer (a PDP 11/23).

Using the computer-controlled, four-roll mill, we were able to investigate drop deformation and burst over a wide range of capillary number, viscosity ratio, and strain-to-vorticity ratio. Data were obtained for flows which had not previously been investigated, and data of improved quality were obtained for pure straining flows which had been studied by previous investigators using manual control of the four-roll mill. An extensive discussion of these results and comparisons with several theories for drop deformation and burst is presented in the second paper in this series (Bentley \& Leal 1986, henceforth referred to as II). In the present paper, we focus on the design of the apparatus and the implementation of the feedback control scheme.

\section{Apparatus}

A schematic of the four-roll mill is shown in figure 1. This shows a top view of four cylindrical rollers which are immersed in a fluid bath. The rollers are rotated to produce a flow field in which the velocity gradient is essentially constant near the device centre. For example, a vorticity-free two-dimensional flow is produced by turning all four rollers at the same speed, but with rollers 2 and 4 in the opposite direction from rollers 1 and 3 , as illustrated in figure 1.

As mentioned above, the four-roll mill is capable of producing two-dimensional flows with an arbitrary strain-rate-to-vorticity ratio. This is accomplished by varying the ratio of the speeds of the crosswise pairs of rollers (i.e. the speeds of rollers 2 and 4 in figure 1 relative to those of rollers 1 and 3). The velocity-gradient tensor near 
the device centre, in a region of dimensions comparable to the gap between the rollers, can be approximated by:

$$
\nabla u=\frac{1}{2} G\left[\begin{array}{ccc}
1+\alpha & 1-\alpha & 0 \\
-1+\alpha & -1-\alpha & 0 \\
0 & 0 & 0
\end{array}\right]
$$

in the Cartesian coordinates of figure 1 (Fuller \& Leal 1981). The flow parameter, $\alpha$, is a measure of the relative strength of the straining motion and vorticity in the flow. It has been reported to be nearly equal to the negative of the ratio of roller speeds 2 and 4 relative to 1 and 3 (Giesekus 1962; Fuller \& Leal 1981), and ranges in value from -1 , corresponding to purely rotational flow, to +1 , corresponding to pure straining motion. The intermediate value, $\alpha=0$, represents simple shear flow. The ratio of the magnitude of the rate of strain tensor to that of the vorticity can be expressed as:

$$
\frac{\text { magnitude of strain rate }}{\text { magnitude of vorticity }}=\frac{1+\alpha}{1-\alpha} \text {. }
$$

In the coordinate system of figure 1 , the velocity $(u, v, w)$ is given by

$$
u=\frac{1}{2} G[(1+\alpha) x+(1-\alpha) y], \quad v=\frac{1}{2} G[(-1+\alpha) x+(-1-\alpha) y], \quad w=0,
$$

with streamlines given by:

$$
(x+y)^{2}-\alpha(x-y)^{2}=c^{2} .
$$

Streamlines for several choices of positive $\alpha$ are shown in figure 2 of II. These are hyperbolas, symmetric about $\theta= \pm{ }_{4}^{1} \pi$ with asymptotes at $\theta=\cos ^{-1}\left[\left(1 \pm \alpha^{\frac{1}{2}}\right) /\right.$ $\left.(2(1+\alpha))^{\frac{1}{2}}\right]$. The principal axes of the rate-of-strain tensor, $\frac{1}{2}\left[\nabla u+(\nabla u)^{\mathrm{T}}\right]$, are at $\theta=0$ and $\theta=\frac{1}{2} \pi$, corresponding to the maxima in extension and compression, respectively. Flows with positive $\alpha$ (so-called strong flows) are of primary interest in the drop deformation and burst problem since the magnitude of the straining rate exceeds the vorticity for $\alpha>0$, and these flows are thus effective in producing appreciable deformation and burst of drops.

The shear rate at the centre of the device, $G$, was found here and in previous studies of the four-roll mill (Giesekus 1962; Fuller \& Leal 1981) to be directly proportional to the speed of the faster pair of rollers, with the constant of proportionality dependent on the geometry of the four-roll mill, but independent of the flow type. The relationship between the roller speeds, flow type, and shear rate for the four-roll mill used in the present study is discussed in $\$ 4$.

The difficulty in controlling the drop position in the four-roll mill can be understood from an inspection of (3), above. In strong flows, there is one stagnation point (at the origin), and it is clearly desirable to position the drop there for ease of observation. However, the stagnation point for the flow field of (3) with $\alpha>0$ is a highly unstable equilibrium position for the drop, as we can easily demonstrate by the following argument. Let us suppose, for simplicity, that a drop will follow the path of a fluid element. Since the velocity gradient (1) is independent of position, the velocity at a point $\boldsymbol{x}$ is given by $\boldsymbol{u}=\boldsymbol{\nabla} \boldsymbol{u} \cdot \boldsymbol{x}$, and thus the drop trajectory can be found by solving the linear system:

$$
\frac{\mathrm{d} x}{\mathrm{~d} t}=\boldsymbol{\nabla} \boldsymbol{u} \cdot \boldsymbol{x}
$$


For $\alpha>0$, this linear system has non-trivial eigenvalues and eigenvectors:

$$
\left.\lambda_{1,2}= \pm G \alpha^{\frac{1}{2}}, \quad \xi_{1,2}=\left[\left(1 \pm \alpha^{\frac{1}{2}}\right) /(2(\alpha+1))^{\frac{1}{2}},\left(-1 \pm \alpha^{\frac{1}{2}}\right) /(2(\alpha+1))^{\frac{1}{2}}, 0\right)\right] .
$$

The presence of the positive eigenvalue indicates that if a drop is initially placed at the origin in a strong flow, any disturbance in its position with a component in the direction of the corresponding eigenvector will grow exponentially. Thus, a drop or particle tends to be carried from the stagnation point in the direction of this eigenvector of the velocity gradient tensor. These eigenvectors correspond to the linear exit streamlines which can be seen in figure 2 of II. For purely extensional flow, $\alpha=1$, the exit streamline is along the $x$-axis, while for flows with $\alpha=0.8,0.6,0.4$, and 0.2 , the exit streamlines are at $\theta_{\mathrm{e}}=-3.2^{\circ},-7.2^{\circ},-12.7^{\circ}$, and $-20.9^{\circ}$ from the $x$-axis, respectively.

Approximate manual control of the drop position is possible in pure straining flow $(\alpha=1)$ because in this particular flow the eigenvectors of (5) are at right-angles to the roller geometry. Thus to keep the drop near the device centre, it is sufficient to adjust the speeds of the left pair of rollers ( 2 and 3 in figure 1) relative to those of the right pair (1 and 4). Even so, this is a difficult task, and all researchers (Taylor 1934 ; Rumscheidt \& Mason 1961 ; Grace 1971) reported problems in keeping the drop at the centre of the apparatus, which limited the useful shear rate range of their apparatus and appears to have resulted in data of only fair reproducibility (due in part to the strong transients introduced into the flow by the manual control process).

While the control problem in pure straining motion is the most severe in the sense that at a given $G$, the speed at which the drop moves away from the stagnation point is greatest (the eigenvalue in (6) is largest), it is less complicated than controlling the drop in a flow with $0<\alpha<1$, because there is only one parameter which must be varied, i.e. the ratio between the speeds of the left and right roller pairs. In the general case, the speed of each roller must be independently varied, a task requiring calculations and coordination beyond the capabilities of an operator. Our interest in the intermediate flow cases was part of the motivation behind the development of the computer controlled four-roll mill.

\section{Four-roll mill design}

\subsection{Flow device}

In the four-roll mill, the flow is well-represented by (1) in a square of dimensions comparable to the width of the gap between adjacent rollers. To accommodate highly deformed drops, we chose $2.54 \mathrm{~cm}$ for the gap width, which is sufficient to allow a draw ratio (length of the deformed drop divided by the undeformed drop diameter) of 25 for a drop with an undeformed diameter of $1 \mathrm{~mm}$, which we felt was the smallest size that would be practical for direct visual observation. The length of the rollers was chosen at $15.5 \mathrm{~cm}$, which proved sufficient to minimize end effects for most of the depth. The dimensions of the tank containing the suspending fluid were fixed at $49.5 \mathrm{~cm}$ square by $17.5 \mathrm{~cm}$ deep. The depth of the tank was adequate to completely immerse the rollers.

The geometry of the device was made adjustable by mounting the rollers on arms which were attached to the base at $45^{\circ}$ to the tank. Thus, the ratio of roller spacing to roller radius could be changed by moving the rollers in or out along these $45^{\circ}$ arms. For purposes of our experiments, the roller spacing to roller radius ratio, $b / r_{\mathbf{r}}$ (see 
figure 1) was fixed at 1.25, a value which results in a good match between the desired hyperbolic streamlines (for $\alpha=1$ ) and the cylindrical roller surface.

The rollers were driven by d.c. stepping motors (Superior Electric M093-FD 301 motors driven by Superior Electric TM600 translator modules). They could be accurately controlled from 250 steps/second to 10000 steps/second, with each step corresponding to $\frac{1}{200}$ of a shaft revolution in full step mode or $\frac{1}{400}$ revolution in half step mode. Typically, the motors were used in half step mode and geared down by a factor of $60: 1$ using 64 pitch double threaded worm gears. This yielded a usable shear rate range of $0.05 \mathrm{~s}^{-1}$ to $2.0 \mathrm{~s}^{-1}$, which proved adequate for our experiments. Other gears with a reduction ratio of $20: 1$ were also purchased for situations where higher shear rates were required. The motors were mounted on rubber and coupled to the shafts using rubber couplings to minimize vibrations.

To minimize the influence of the bottom of the tank, the suspending fluid was floated on a thin layer of mercury. The large density difference between the test fluid and the mercury kept the deformation of the interface between the two insignificant. Since the control scheme depended on backlighting the drop, a small $(4 \mathrm{~cm}$ square $\times 0.64 \mathrm{~cm}$ thick) Plexiglas window was glued to the bottom of the tank, protruding above the mercury layer about $2 \mathrm{~mm}$. The edges of the window were concave to conform to the hyperbolic flow field $(\alpha=1)$, and it was aligned with its corners pointing along the $x$ - and $y$-axes. A similarly shaped but somewhat larger piece of Plexiglas was suspended several millimetres into the fluid from the top surface, which was otherwise open. This was necessary to allow lighting from the top without the distortion which would have resulted from the deformation of a free surface. A slight secondary flow remained despite the mercury layer, with flow upward near the top surface and downward near the bottom. Also, the drop and suspending fluid densities could not be exactly matched, so there was also some vertical motion of the drop due to sedimentation. Neither of these problems proved serious, and it was found that for the shear rates needed for the experiments, the drop could be kept in the central $8 \mathrm{~cm}$ in the vertical direction for up to 15 minutes, which proved to be adequate for the experiment.

\subsection{Lighting and photography}

The illumination of the flow field was very important to the success of the experiment, since high contrast was required for the imaging process to successfully differentiate between the drop and the background. The central portion of the flow field was illuminated by a collimated beam of light from a point source (Oriel Model 6340). The beam of light, collimated with a $75 \mathrm{~mm}$ lens, was passed through the flow field and converged onto the light sensitive array of the video camera using a $105 \mathrm{~mm}$ lens and an $80 \mathrm{~mm}$ microscope objective. This technique yielded a shadowgraph image, quite sensitive to refractive index differences in the field. The drops thus showed up as dark in the bright background.

In order to take pictures of the drop simultaneously with video and still cameras, a cubic beam splitter, $3.8 \mathrm{~cm}$ on a side, was inserted above the focusing lens of the video camera, and the still camera mounted at $90^{\circ}$ from the vertical axis. Both cameras were initially focused on the same point in the flow field, and subsequent focusing during the course of the experiment was accomplished by moving both cameras and the beam splitter vertically as a unit, using a motorized measuring microscope mount specifically adapted for this purpose. The still camera was a Canon A-1 with Macro Lens FD200mm $f / 4.0$ and $50 \mathrm{~mm}$ extension tube, yielding a 
magnification of 1.34 life size with a working distance of about $40 \mathrm{~cm}$. Kodak Tri-X Pan 400 ASA film was used with an $f$-stop of 4.0 and an exposure time of $\frac{1}{15}$ or $\frac{1}{30}$ second.

\subsection{Camera-computer interface}

The video camera used in the experiments was a General Electric model TN2500, a solid-state charge injection device (CID) closed-circuit television camera. The sensor in the camera is an array of $248 \times 244$ discrete picture elements, or pixels. These are sequentially scanned by the circuitry in the camera, which produces two outputs, an analog signal in standard broadcast format, which was directed to a video monitor, and an eight-bit digital representation of the intensity of light at each pixel as it is scanned. A difficulty in effecting control based on a video image is the sheer volume of information in a single image. For example, with the camera we used, each pixel in the field is scanned 60 times each second, resulting in a data rate of 3.36 million bytes per second. This is far faster than can be handled by the current generation of laboratory minicomputers such as the Digital Equipment Corporation PDP 11/23 used in our experiment, even using direct memory access (DMA) transfer. Fortunately, in our case, the signal processing required was extremely simple. Since we were concerned only with the position of the centre of mass of a single drop in the flow, and the lighting could be arranged so that there was high contrast between the drop and the background, it was sufficient to 'threshold' the video signal. This was done in a pipelined manner using a specially designed interface, which compared the incoming intensity to a programmable threshold, yielding a 'one' where the intensity was below the threshold and a 'zero' where the intensity was above this threshold. The incoming data were thus reduced to one output bit for each eight-bit input. Those were assembled into 16-bit words and sent in parallel through a direct memory access interface to the computer. This reduced the net data rate to $0.453 \mathrm{MHz}$, which is just within the limits of the PDP 11/23. The thresholded picture was also made available from the interface in broadcast format, so that it could be displayed on a second video monitor. This was invaluable in setting the threshold value.

Standard broadcast format is 'interlaced', meaning that all the odd-numbered lines of information are transmitted sequentially, followed by the even-numbered lines. This is done to prevent a noticeable flicker when viewing a television. Display time for each 'field' is $\frac{1}{60} \mathrm{~s}$. In controlling the drop, accurate determination of the position in the $x$-direction was more important than that in the $y$-direction, since the exit streamline was aligned closer to the $x$-axis than to the $y$-axis. Thus, only one field of information was used to determine the drop centre of mass, giving a resolution of 248 in the $x$-direction and 122 in the $y$-direction. The field selected (odd or even) depended on which started soonest after the computer's command to 'grab' a frame of picture information. The time necessary to get the frame was generally less than $\frac{1}{60} \mathrm{~s}$, since the size of the 'window' for which thresholded information was to be sent could be adjusted by the operator. Generally, only a fairly small fraction of the screen was covered by the drop, and the window was set accordingly.

Once a frame of information was passed into the computer's memory, the next step was to determine the centre of mass of all the dark (one) bits. This was done using a straightforward routine written in PDP/11 assembly language for speed. This step took between $\frac{1}{20} \mathrm{~s}$ and $\frac{1}{6} \mathrm{~s}$ depending on the window size and the picture composition. The elapsed time for the centre of mass calculation was accurately measured using a timer interface in the PDP 11/23.

The sequence of getting a frame of information and finding the drop centre of mass comprised the measurement portion of the feedback control process. 


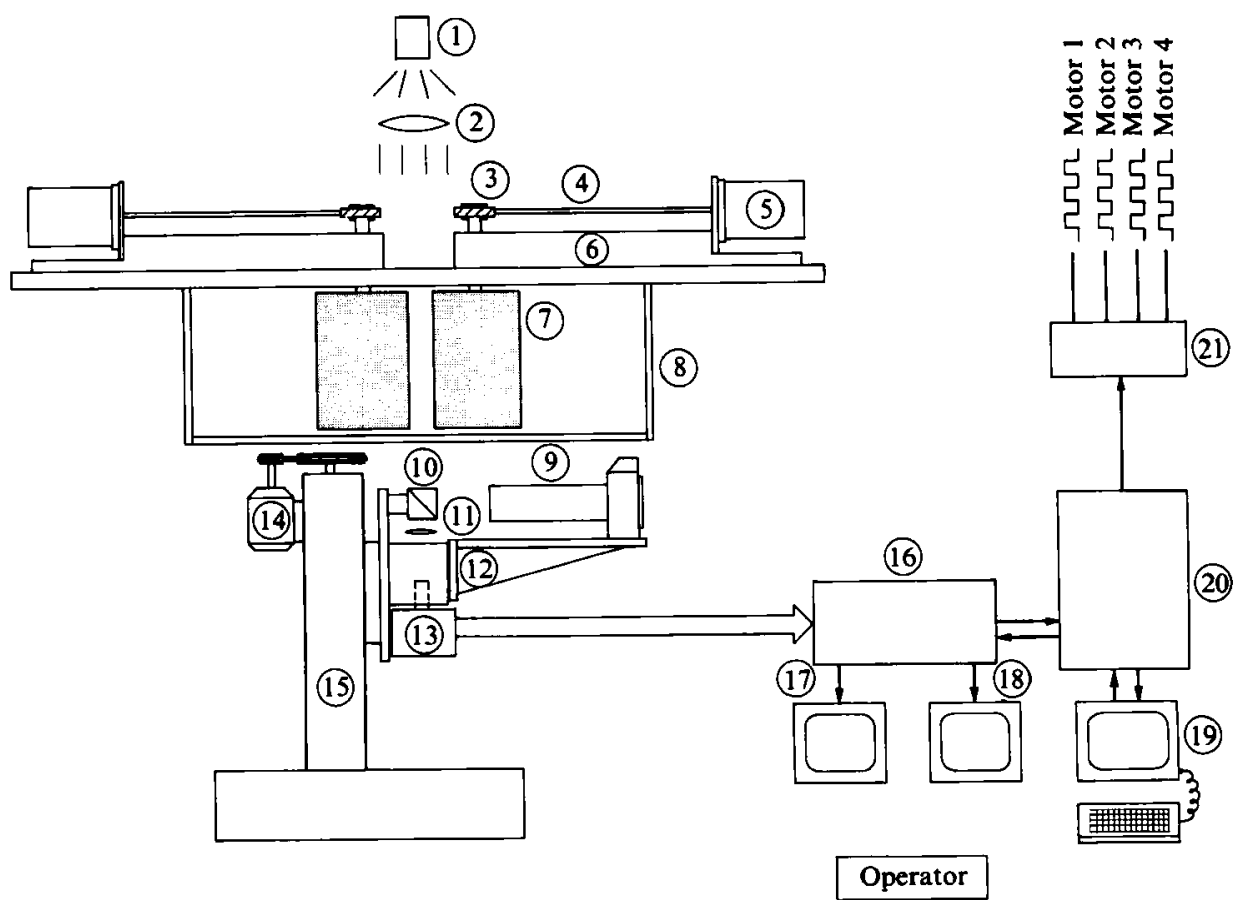

Fiaure 2. Side-view schematic of four-roll mill. (1) Point light source; (2) collimating lens; (3) worm gears; (4) drive shafts; (5) d.c. stepping motors; (6) roller mounting arms; (7) rollers; (8) glass tank; (9) still camera; (10) cubic beam splitter; (11) converging lens; (12) still-camera mount; (13) digital video camera; (14) motor for moving camera assembly vertically; (15) modified measuring microscope mount; (16) video preprocessor; (17) monitor for viewing unprocessed video; (18) monitor for viewing threshold video; (19) video terminal; (20) DEC minicomputer; (21) Apple microcomputer.

\subsection{Computer-stepping motor interface}

In order to effect control of the flow field, the computer had to regulate the motor speeds. Stepping motors are ideal for precise digital control since one need only generate square-wave voltage signals of frequency appropriate for the desired motor speed. An Apple II + microcomputer was programmed to generate these signals and interfaced to the stepping motor translators which energize the motor windings in the proper sequence to turn the shafts. The Apple was interfaced to the DEC computer using a standard RS232-C serial line run at 19200 baud. The DEC computer calculated the desired motor speeds and transmitted these to the Apple for implementation. When the motors speeds were changed, the Apple generated frequency signals ramped from the old speed to the new, as attempts to change speeds too abruptly caused the stepping motors to stall. The Apple was capable of generating frequencies from 20 to $10000 \mathrm{~Hz}( \pm 0.1 \%)$ on each of four independent channels. These signals were sent to the inputs of the four stepping motor translators and this set-up functioned as the actuator in the control process.

Figure 2 shows a side-view schematic of the entire four-roll mill plus computer that was used in the experiment. 


\section{Control of the four-roll mill}

\subsection{Control scheme}

Successful drop-deformation and burst experiments require that the drop be kept as close as possible to the centre stagnation point. The only means of controlling the drop position is by changing the flow field through adjustments to the roller speeds. These changes must be as small as possible to avoid significant disruption to the characteristics of the flow field under study, i.e. the shear rate and flow type. Thus our control objectives are to keep the drop as close to the device centre as possible while minimizing the changes of the 'uncontrolled' flow field.

When the drop drifts away from the origin, alterations to the flow field must be such that the new flow tends to return the drop to the centre of the device. For the case where $\alpha=1.0$, the required response is obvious. If, for example, the drop drifts to the right, the left pair of rollers is speeded up and the right pair slowed down in proportion. This has the effect of superimposing a translational motion (right to left) on the hyperbolic flow field, moving the point of zero flow (stagnation point) to the right. It is clear that for the drop to tend to return to the centre, it must be at a point in the new flow where the net velocity is towards the origin, which requires that the stagnation point be further from the origin than the centre of mass of the drop. The same reasoning applies for any strong flow, with the only complication being that for $0<\alpha<1$ the drop moves along the exit streamline which is at an angle to the roller geometry, requiring a response with changes to all four roller speeds. In the discussion which follows, we consider the stagnation-point position to be the control variable which is regulated by adjustments to the roller speeds, but we could alternatively consider the control variable to be the translational velocity superimposed on the flow field by roller speed changes. The two points of view are equivalent, but the former proves more convenient since the stagnation-point position is more easily measured when calibrating the four-roll mill, so a required control action (stagnation point movement) is more easily translated into the corresponding roller speed changes.

The sequence of events in our control scheme is as follows. The computer requests a frame of thresholded information concerning the light intensity at the pixels within the 'window'. This information is stored in the computer's memory via direct memory access from the custom built camera-computer interface. When the window is complete, the processor is signalled that the information is ready for processing. Elapsed time for this step is $0.01-0.05 \mathrm{~s}$. The computer calculates the centre of mass of the drop. Elapsed time is $0.05-0.15 \mathrm{~s}$. The centre of mass is used to calculate the required control action and corresponding roller speeds from the control model, taking about $0.01 \mathrm{~s}$. The DEC computer transmits the new speeds to the Apple microcomputer which ramps the motors from their previous speeds to the new ones. This step takes about $0.05 \mathrm{~s}$. The computer checks to see if there is any operator input to change the threshold value, the window size, or the shear rate, and makes requested adjustments. The process is then repeated. Typically, between five and ten control cycles take place each second.

Calculation of appropriate roller speeds from the drop-position data requires a control model describing the flow field's response to roller speed changes and the drop's response to flow-field changes. A rigorous description would require a solution of the equations of motion throughout the experimental device, including the region inside the deformed drop. Since this was clearly not possible in a real-time control 
scheme, a simple, approximate model was developed. In formulating this model we assumed that the drop velocity is equivalent to that of a fluid element placed at its centre of mass in the undisturbed flow. Since the drop experiences no external forces except gravity, and we are considering the case where the drops are neutrally buoyant, this approximation becomes exact as the drop size approaches zero (and proved empirically to be an adequate approximation even when the drop is quite deformed). We also assumed that the stagnation point could be moved to any position near the origin (through suitable roller-speed selection) without affecting the flow type or shear rate. This assumption is plausible since we are concerned only with small departures from the uncontrolled flow. The actual selection of the roller speeds and the verification of this assumption is discussed below. Finally, we assumed that the speed of movement of the stagnation point is proportional to its distance from the 'set' point. The set point is where the stagnation point would end up if the roller speeds were held at the new speeds indefinitely. The constant of proportionality, $1 / \tau$, is expected to depend on the kinematic viscosity of the suspending fluid. Since we are considering only small displacements of the stagnation point, this last assumption can be considered as a linearization of the actual stagnation point motion. These assumptions result in the following simple model:

$$
\begin{aligned}
2 \frac{\mathrm{d} x}{\mathrm{~d} t} & =(1+\alpha)\left(x-x_{\mathrm{s}}\right)+(1-\alpha)\left(y-y_{\mathrm{s}}\right), \\
2 \frac{\mathrm{d} y}{\mathrm{~d} t} & =(-1+\alpha)\left(x-x_{\mathrm{s}}\right)+(-1-\alpha)\left(y-y_{\mathrm{s}}\right), \\
\frac{\mathrm{d} x_{\mathrm{s}}}{\mathrm{d} t} & =\frac{1}{\tau}\left(x_{\mathrm{ss}}-x_{\mathrm{s}}\right), \quad \frac{\mathrm{d} y_{\mathrm{s}}}{\mathrm{d} t}=\frac{1}{\tau}\left(y_{\mathrm{ss}}-y_{\mathrm{s}}\right),
\end{aligned}
$$

where $(x, y)$ is the drop position, $\left(x_{\mathrm{s}}, y_{\mathrm{s}}\right)$ is the stagnation point position and $\left(x_{\mathrm{ss}}, y_{\mathrm{ss}}\right)$ is the stagnation point set point, which is the control variable. All times are rendered dimensionless with respect to $G^{-1}$ and all distances with respect to $b$, the distance from the centre of the device to the line joining the centres of adjacent rollers (see figure 1). According to our model, the system is non-interacting (the drop position has no effect on the stagnation point), so the latter pair of equations can be solved immediately for $\left(x_{\mathrm{s}}, y_{\mathrm{s}}\right)$. We are thus led to consider the system:

$$
\frac{\mathrm{d} \boldsymbol{x}}{\mathrm{d} t}=\boldsymbol{A} \cdot \boldsymbol{x}-\boldsymbol{A} \cdot \boldsymbol{b}
$$

where

$$
\boldsymbol{x}=\left[\begin{array}{l}
x \\
y
\end{array}\right], \quad \boldsymbol{A}=\frac{1}{2}\left[\begin{array}{rr}
1+\alpha & 1-\alpha \\
-1+\alpha & -1-\alpha
\end{array}\right], \quad \boldsymbol{b}=\left[\begin{array}{l}
x_{\mathrm{s}} \\
y_{\mathrm{s}}
\end{array}\right] .
$$

Since our model assumes that the entire flow field can be moved about within the four-roll mill, and that the drop follows the fluid trajectories in this translated flow, it is obvious that the homogeneous portion of this linear system is exactly the same as that for the uncontrolled flow given by (5). Thus the eigenvalues and eigenvectors are identical (apart from the non-dimensionalization by the shear rate):

$$
\left.\lambda_{1,2}= \pm \alpha^{\frac{1}{2}}, \quad \xi_{1,2}=\left[\left(1 \pm \alpha^{\frac{1}{2}}\right) /(2(\alpha+1))^{\frac{1}{2}},\left(-1 \pm \alpha^{\frac{1}{2}}\right) /(2(\alpha+1))^{\frac{1}{2}}, 0\right)\right] .
$$

We note that the component of a disturbance along the eigenvector with a negative eigenvalue will decay, so we are concerned only with movement along the eigenvector 


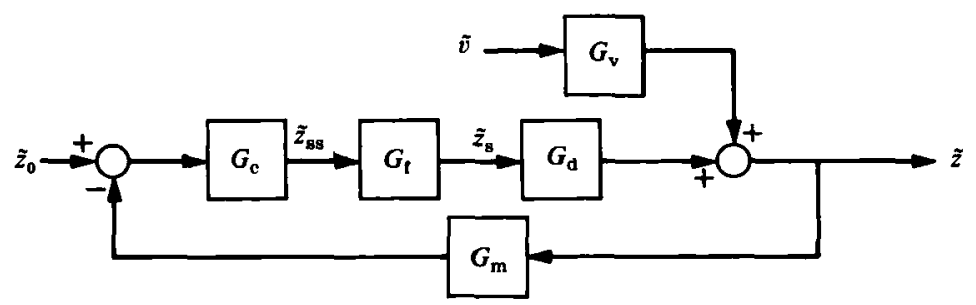

FiguRE 3. Block diagram for proportional control of drop position.

corresponding to the positive eigenvalue. Thus, if we define $z$ to be the distance from the origin along this eigenvector:

$$
x=z\left(\alpha^{\frac{1}{2}}+1\right) /(2(\alpha+1))^{\frac{1}{2}}, \quad y=z\left(\alpha^{\frac{1}{2}}-1\right) /(2(\alpha+1))^{\frac{1}{2}},
$$

then substituting into the homogeneous portion of $(9)$ gives:

$$
\frac{\mathrm{d} z}{\mathrm{~d} t}=\alpha^{\frac{1}{2} z}
$$

reducing the problem to one dimension. We further note that if we maintain the roller speeds such that $\left(x_{\mathrm{ss}}, y_{\mathrm{ss}}\right)$ is always along the eigenvector with displacement $z_{\mathrm{ss}}$, then $\left(x_{\mathrm{s}}, y_{\mathrm{s}}\right)$ will also be on the eigenvector with displacement $z_{\mathrm{s}}$, and (8) gives:

$$
\frac{\mathrm{d} z_{\mathrm{s}}}{\mathrm{d} t}=\frac{1}{\tau}\left(z_{\mathrm{ss}}-z_{\mathrm{s}}\right)
$$

Thus the system of (7) and (8) can be written

$$
\begin{aligned}
& \frac{\mathrm{d} z}{\mathrm{~d} t}=\alpha^{\frac{1}{2}}\left(z-z_{\mathrm{s}}\right), \\
& \frac{\mathrm{d} z_{\mathrm{s}}}{\mathrm{d} t}=\frac{1}{\tau}\left(z_{\mathrm{ss}}-z_{\mathrm{s}}\right) .
\end{aligned}
$$

Our two objectives in the design of the control algorithm are to keep the drop displacement $z$ and the stagnation point displacement $z_{\mathrm{s}}$ as small as possible.

Our actual computer based control sequence is best described as a discrete sampled system with a time-lag corresponding to the sum of the times required to obtain the frame information, determine the drop position, and calculate the new roller speeds. Nevertheless, it is instructive to first consider the system as a classical continuous controller with a measurement delay (c.f. Douglas 1972). Figure 3 shows a block diagram of the system in the Laplace domain. Here $G_{\mathrm{c}}$ is the controller transfer function, $G_{\mathrm{p}}$ the fluid transfer function representing the stagnation-point movement described by (16), and $G_{d}$ the drop transfer function representing the response of the drop to the flow field given by (15). $G_{\mathrm{v}}$ is the transfer function of the response to an unspecified disturbance $\tilde{v}$. In addition, $\tilde{z}_{0}$ is the desired drop position (here equal to 0 , since we want the drop to be at the origin), $\tilde{z}_{\mathrm{ss}}$ the stagnation point set, and $\tilde{z}_{\mathrm{s}}$ the stagnation point. $G_{\mathrm{m}}$ is the transfer function representing the measurement delay. In the present case,

$$
\begin{aligned}
G_{\mathrm{f}}(s) & =\frac{1}{1+\tau s}, \\
G_{\mathrm{d}}(s) & =\frac{1}{1-\alpha^{-\frac{1}{2} s}},
\end{aligned}
$$


and for simple proportional control,

$$
G_{\mathrm{c}}(s)=K_{\mathrm{c}} .
$$

The measurement delay $G_{\mathrm{m}}(s)$ is exactly represented in the Laplace domain by

$$
G_{\mathrm{m}}(s)=\mathrm{e}^{-t_{\mathrm{d}} s}
$$

However, to simplify the analysis, we use the well-known Pade approximation for the delay transfer function,

$$
G_{\mathrm{m}}(s) \approx \frac{1-\frac{1}{2} t_{\mathrm{d}} s}{1+\frac{1}{2} t_{\mathrm{d}} s}
$$

From the block diagram,

$$
\tilde{z}(s)=\frac{G_{0}}{1+G_{0} G_{\mathrm{m}}} \tilde{z}_{0}(s)+\frac{G_{\mathrm{v}}}{1+G_{0} G_{\mathrm{m}}} \tilde{v}(s),
$$

with

$$
G_{0}(s)=-K_{\mathrm{c}} G_{\mathrm{f}} G_{\mathrm{d}} \text {. }
$$

The behaviour of the controller is determined by the roots of $1+G_{0} G_{\mathrm{m}}$, each of which must have a negative real part for the system to be stable. A Routh array was used to determine conditions where the real part of roots were negative, yielding the following stability criteria:

$$
\begin{gathered}
K_{\mathrm{c}}>1, \\
\tau<\alpha^{-\frac{1}{2}}, \\
t_{\mathrm{d}}<\frac{\alpha^{-\frac{1}{2}}-\tau-\frac{2 \tau \alpha^{-\frac{1}{2}} K_{\mathrm{c}}}{\alpha^{-\frac{1}{2}}-\tau}+\left[\left(\alpha^{-\frac{1}{2}}-\tau\right)^{2}+\left(\frac{2 \tau \alpha^{-\frac{1}{2}} K_{\mathrm{c}}}{\alpha^{-\frac{1}{2}}-\tau}\right)^{2}+4 \tau \alpha^{-\frac{1}{2}}\right]^{\frac{1}{2}}}{1+K_{\mathrm{c}}} .
\end{gathered}
$$

The requirement of (24) that the controller gain be greater than unity simply means that the stagnation point must be moved further from the origin than the drop in order to move the drop in the correct direction (as we anticipated). The second criterion specifies that the response of the fluid to changes in the roller speed must be fast compared to the shear rate (recall that $\tau$ is relative to $G^{-1}$ ). This limits the shear rate for which the drop is controllable with this simple control scheme. Since the response time of a fluid is inversely proportional to its kinematic viscosity, we would expect a proportional control scheme to be more successful with a more viscous suspending fluid. These two criteria are independent of the presence of the measurement delay, $t_{\mathrm{d}}$.

The third stability requirement illustrates the destabilizing effect of the measurement delay. This places a greater restriction on the controllable shear-rate range of the device, with the destabilizing effect magnified as the gain increases. Figure 4 shows the stable region of $(24)-(26)$ in the $\left(\tau, t_{\mathrm{d}}\right)$-plane, with $K_{\mathrm{c}}$ as a parameter for hyperbolic flow $(\alpha=1)$.

From the analysis above, it is clear that control schemes based on conventional continuous controllers are not optimal for control of drops in a four-roll mill owing to the finite response time of the fluid and the measurement delay in locating the drop. This was verified by computer simulations of proportional control. These simulations were performed by starting the drop at a fixed displacement from the origin, and calculating the drop and stagnation-point positions based on the model above, with the stagnation-point set point computed from the simple proportional control scheme. The measurement time, non-dimensionalized by the shear rate, was 


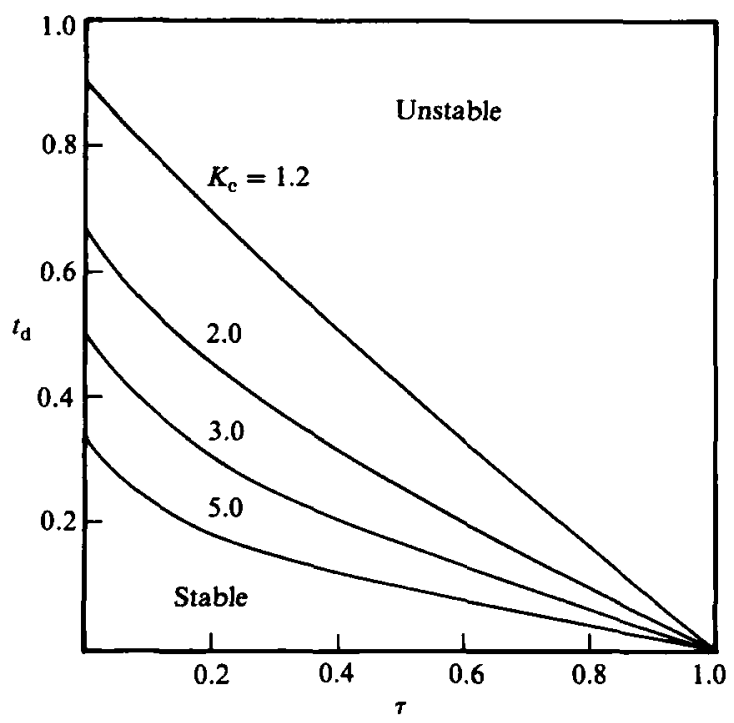

FIGURE 4. Stability diagram for simple proportional control.

chosen in the range from 0.2 to 1.0 , corresponding to shear rates from about 0.7 to 3.5 given the 'worst case' measurement delay encountered in getting the thresholded image from the camera and computing the centre of mass. Various values for $\tau$, the response time of the fluid, were tried. The proportional control scheme was successful only for low values of the measurement delay. When the $t_{\mathrm{d}}$ was larger than about 0.5 , the controller failed no matter what value of the gain, $K_{c}$, was chosen. With a small gain (e.g. 1.2), the controller tended to 'chase' the drop as it went further and further from the origin, since the drop was moving exponentially from the stagnation point during the time delay, while the simple proportional controller was correcting linearly, and thus consistently underestimating the required control action. If the gain was increased (e.g. 2.5), the controller overshot the required response and caused the drop (and stagnation point) to oscillate around the origin in an unacceptable manner. As expected, both types of behaviour were exacerbated by slow fluid response (large $\tau$ ). Similar simulations using proportional-derivative control were performed, but proved no more successful. In practice, the derivative mode would have required differentiation of the drop position data as well, which would have been an inherently inaccurate calculation. Moreover, both control schemes were very sensitive to inaccuracies in the drop position data.

These simulations showed that a simple continuous scheme would have been very difficult or impossible to successfully implement. Also, since the apparatus incorporates a computer as part of the position sensor, the calculations necessary to implement a more-sophisticated control scheme do not present an obstacle. We thus developed an inferential control scheme which used the model described above to extrapolate the drop movement from the position data, known stagnation point history, and measurement delay.

The most obvious strategy would be to set the stagnation point to move the drop position some fraction of its displacement towards the origin in some period of time, with the most natural period being that of the control cycle. In this case, the measurement delay is the slow step, so the time for a complete control cycle can be 
approximated by $t_{\mathrm{d}}$. If $z_{i}, z_{\mathrm{s}, i}, z_{\mathrm{ss}, i}$ are the values of $z, z_{\mathrm{s}}, z_{\mathrm{ss}}$, at the end of control cycle $i$, then solution of (15) and (16) yields:

$$
\begin{gathered}
z_{i+1}=\mathrm{e}^{\left(\alpha^{\frac{1}{2}} t_{\mathrm{d}}\right)} z_{i}+z_{\mathrm{ss}, i}\left(1-\mathrm{e}^{\left(\alpha^{\frac{1}{2}} t_{\mathrm{d}}\right)}+\frac{\alpha^{\frac{1}{2}} \tau}{1+\alpha^{\frac{1}{2} \tau}}\left(z_{\mathrm{s}, i}-z_{\mathrm{ss}, i}\right)\left(\mathrm{e}^{-t_{\mathrm{d}} / \tau}-\mathrm{e}^{\alpha^{\frac{1}{2}} t_{\mathrm{d}}}\right)\right), \\
z_{\mathrm{s}, i+1}=z_{\mathrm{ss}, i}+\left(z_{\mathrm{s}, i}-z_{\mathrm{ss}, i}\right) \mathrm{e}^{-t_{\mathrm{d}} / \tau}
\end{gathered}
$$

and the control strategy requires

$$
z_{i+1}=(1-r) z_{i}
$$

which, upon substitution of (28) and (29) into (27) gives the new stagnation set point as:

$$
z_{\mathrm{ss}, i}=\frac{\left(1-r-\mathrm{e}^{\left(\alpha^{\frac{1}{2}} t_{\mathrm{d}}\right)} z_{i}-\frac{\alpha^{\frac{1}{2}} \tau}{1+\alpha^{\frac{1}{2} \tau}}\left(\mathrm{e}^{-t_{\mathrm{d}} / \tau}-\mathrm{e}^{\left(\alpha^{\frac{1}{2}} t_{\mathrm{d}}\right)} z_{\mathrm{s}, i}\right.\right.}{1-\frac{1}{1+\alpha^{\frac{1}{2} \tau}} \mathrm{e}^{\left(\alpha^{\frac{1}{2}} t_{\mathrm{d}}\right)}-\frac{\alpha^{\frac{1}{2} \tau}}{1+\alpha^{\frac{1}{2} \tau}} \mathrm{e}^{-t_{\mathrm{d}} / \tau}}
$$

Note that for a given control cycle $i$, we measure $z_{i-1}$ and compute $z_{i}$ from (27) written for $i-1$ and the known stagnation-point history. We then calculate $z_{\mathrm{ss}, i}$ from (30).

Simulations of this control scheme, similar to those described above for simple proportional control, showed that it too was unsatisfactory. The drop moved closer to the origin at the end of each control cycle (as we require), but movement during each control cycle was oscillatory as was the movement of the stagnation set point. These oscillations were damped with the damping depending on the ratio $\tau / t_{\mathrm{d}}$. For $\tau / t_{\mathrm{d}}$ greater than one, the oscillations grew with time, so this control scheme was also rejected.

A second approach was to bring the drop to the device centre in a more orderly fashion by requiring that the velocity of the drop be proportional to its displacement from the origin at the end of each control cycle. This is equivalent to specifying the relative positions of the drop and the stagnation point:

$$
z_{\mathrm{s}, i+1}-z_{i+1}=r z_{i+1} \text {. }
$$

Substituting (31) and (28) into (27) yielded for the stagnation-point set point:

$$
z_{\mathrm{ss}, i}=\frac{(r+1) \mathrm{e}^{\alpha^{\frac{1}{2}} t_{\mathrm{d}}} z_{i}+\left((r+1) \frac{\alpha^{\frac{1}{2} \tau}}{1+\alpha^{\frac{1}{2}} \tau}\left(\mathrm{e}^{-t_{\mathrm{d}} / \tau}-\mathrm{e}^{\left(\alpha^{\frac{1}{2}} t_{\mathrm{d}}\right)}\right)-\mathrm{e}^{-t_{\mathrm{d}} / \tau}\right) z_{\mathrm{s}, i}}{1-\mathrm{e}^{-t_{\mathrm{d}} / \tau}-(r+1)\left(1-\frac{1}{1+\alpha^{\frac{1}{2} \tau}} \mathrm{e}^{\left(\alpha^{\frac{1}{2}} t_{\mathrm{d}}\right)}-\frac{\alpha^{\frac{1}{2} \tau}}{1+\alpha^{\frac{1}{2} \tau}} \mathrm{e}^{-t_{\mathrm{d}} / \tau}\right)} .
$$

Simulations of this control scheme showed it to be much better behaved. The drop and the stagnation point moved monotonically to the centre of the device without oscillations for any ratio $\tau / t_{\mathrm{d}}$.

This latter control scheme was implemented for the experiment. Tests of the control were made using a neutrally buoyant solid particle. The results were quite satisfying. Once the control scheme parameters were properly selected (in particular, the scheme was sensitive to an accurate estimate for the value of $\tau$, the response time of the fluid), a particle could be maintained within a millimetre of the device centre for shear rates up to $5 \mathrm{~s}^{-1}$ in flows with $0.2 \leqslant \alpha \leqslant 1.0$, provided that the origin of the flow field (where the stagnation point would be in the absence of any control action) was initially aligned with the origin of the camera (where the computer would 
compute a centre of mass of $(0,0))$. Otherwise, the misalignment would cause a systematic error in the centre of mass determination, resulting in a slow drift of the particle away from the origin, as its displacement and the required control action would be consistently underestimated. To overcome this difficulty, an integral control mode was added which became active only when the particle was more than a specified distance from the camera centre. This ensured that the particle did not stray far from the centre of the flow field and gave the operator plenty of time to correct any misalignment by moving the entire camera assembly in the $x$-direction. Any misalignment in the $y$-direction was far less critical (since the maximum angle of the exit streamline was inclined only $21^{\circ}$ from the $x$-axis for $\alpha=0.2$ ). Provisions were made for aligning the origin in the $y$-direction in software. With a little practice, the symptoms of this misalignment were easily recognized and appropriate corrections made.

No facility was available to attempt hand control of the drop, but indications from previous researchers (Taylor 1934; Rumscheidt \& Mason 1961; Grace 1971) were that manual control was possible for shear rates no higher than $0.7 \mathrm{~s}^{-1}$, and then, of course, the flow was limited to irrotational flow. Thus, the computer-based control scheme developed here was much superior, both in allowing larger shear rates and all flow types between $0.2 \leqslant \lambda \leqslant 1$. Comparison with a film of Grace's (1971) experiments suggests that the present computer-based scheme was also superior in that it was able to detect and correct movements of the drop away from the centre of the device before they were large enough to be recognized by eye, so that required changes in the flow were imperceptible visually.

In the drop deformation experiments described in II, the drop size, interfacial tension, suspending fluid viscosity, and viscosity ratio were such that drop burst usually occurred for shear rates less than $0.7 \mathrm{~s}^{-1}$, leaving a substantial overcapacity in the controller. Under these conditions, the drop and the stagnation point were maintained within $0.05 \mathrm{~cm}$ of the device centre at all times, and the control scheme was considered successful.

\subsection{Stagnation-point position}

In developing our control scheme, we assumed that the stagnation point in the flow could be moved within the device without upsetting the shear rate or the flow type significantly. In this section we propose a simple model which will allow calculation of the four roller speeds necessary to generate a flow with a given position for the stagnation point and a given (fixed) shear rate, and flow type. This model has been tested and found adequate for small displacements of the stagnation point from the origin.

In the uncontrolled four-roll mill flow, the flow type is determined by the ratio of the speeds of the two diagonal pairs of rollers, and the shear rate is governed by the speed of the faster pair. Since the stagnation point is at the centre, the speeds of diagonally opposed rollers are always equal. When the stagnation point is to be moved, the speeds of diagonal pairs are no longer identical, so some generalization of these relations between roller speed and shear rate is needed.

We start by assuming that in the case where the speeds of the faster pair of rollers, $\omega_{1}$ and $\omega_{3}$, are unequal, the shear rate can be kept constant by fixing the sum of their speeds, with the required sum depending on the desired shear rate:

$$
\omega_{1}+\omega_{3}=f_{1}(G) \text {. }
$$




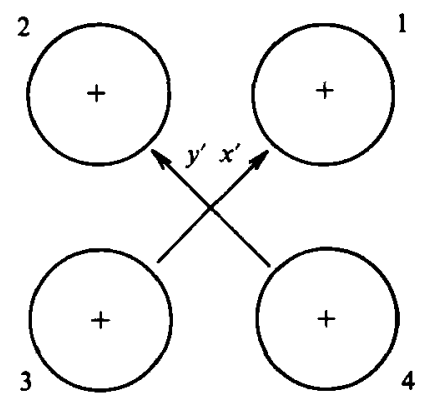

Figure 5. Schematic of four-roll mill with rotated coordinate system.

Similarly, we assume that a given flow type can be generated by fixing the ratio of the sum of the speeds of diagonally opposed rollers. The required ratio depends on the flow type to be generated:

$$
-\frac{\omega_{2}+\omega_{4}}{\omega_{1}+\omega_{3}}=f_{2}(\alpha)
$$

The minus sign appears because rollers 2 and 4 are turned in the opposite direction from rollers 1 and 3 for $\alpha>0$.

We further propose that the position of the stagnation point along the line joining rollers one and three depends only on the ratio of the speeds of these two rollers and similarly for rollers two and four:

$$
\begin{aligned}
& \frac{\omega_{3}}{\omega_{1}}=f_{3}\left(x_{\mathrm{s}}^{\prime}\right), \\
& \frac{\omega_{4}}{\omega_{2}}=f_{4}\left(y_{\mathrm{s}}^{\prime}\right) .
\end{aligned}
$$

Here $\left(x_{\mathrm{s}}^{\prime}, y_{\mathrm{s}}^{\prime}\right)$ is the position of the stagnation point in the $\left(x^{\prime}, y^{\prime}\right)$-coordinate system of figure 5 .

In order to achieve a given desired flow type, shear rate, and stagnation point, $f_{1}$, $f_{2}, f_{3}$ and $f_{4}$ must be determined from the calibration data, and the roller speeds $\omega_{1}$, $\omega_{2}, \omega_{3}$ and $\omega_{4}$ calculated by solving the four equations above. These form the linear system :

$$
\left[\begin{array}{cccc}
1 & 0 & 1 & 0 \\
f_{2} & 1 & f_{2} & 1 \\
-f_{3} & 0 & 1 & 0 \\
0 & -f_{4} & 0 & 1
\end{array}\right]\left[\begin{array}{l}
\omega_{1} \\
\omega_{2} \\
\omega_{3} \\
\omega_{4}
\end{array}\right]=\left[\begin{array}{l}
f_{1} \\
0 \\
0 \\
0
\end{array}\right]
$$

which has the solution:

$$
\begin{aligned}
& \omega_{1}=\frac{f_{1}}{\left(f_{3}+1\right)}, \\
& \omega_{2}=-\frac{f_{1} f_{2}}{\left(f_{4}+1\right)},
\end{aligned}
$$




$$
\begin{aligned}
& \omega_{3}=\frac{f_{1} f_{3}}{\left(f_{3}+1\right)}, \\
& \omega_{4}=-\frac{f_{1} f_{2} f_{4}}{\left(f_{4}+1\right)} .
\end{aligned}
$$

Calibration experiments described in the next section were performed to test the assumptions which lead to $(33)-(36)$, and thereby to $(38)-(41)$. These experiments demonstrate that the approximations (33)-(36) are adequate for small displacements of the stagnation point from the device centre. The functions $f_{1}$ to $f_{4}$ were also determined from these calibration experiments.

\subsection{Flow-field calibration}

In order to relate the flow-field characteristics to the roller speeds, the flow in the four-roll mill was investigated photographically. This was necessary to determine the following: the precise flow type, $\alpha$, for a given ratio of the speeds of rollers 2 and 4 relative to those of rollers 1 and 3 [i.e. the function $f_{2}$ of (34) with the stagnation point in the centre]; the relationship between the rollers speeds and the shear rate [i.e. the function $f_{1}$ of (33)]; and the position of the stagnation point in the device as a function of the roller speeds [i.e. the functions $f_{3}$ and $f_{4}$ of (35) and (36)].

To visualize the streamlines in the flow, the four-roll mill was filled with Chevron Polybutene 16 laced with a low concentration of Emerson and Cummings Ecosphere Microballoons, hollow glass spheres of $30-50 \mu \mathrm{m}$ diameter, which were highly reflective and served as tracer particles. The flow field was illuminated from two sides with thin $\left(\sim \frac{1}{2} \mathrm{~cm}\right)$ horizontal planes of light produced with projector bulbs shone through a deep slit formed from two parallel aluminium plates held $0.3 \mathrm{~cm}$ apart. These slits were positioned midway between the top and bottom of the tank. Time exposure pictures were taken from below the tank at a lens opening of $f / 3.5$ for periods ranging from $\frac{1}{4}$ to $1 \mathrm{~s}$.

The flow type parameter, $\alpha$, was easily determined from macroscopic features of the streamlines of the flow given by (4). For $\alpha>0$ the dividing streamlines, found by taking $c^{2}=0$ in (4), are linear, corresponding to the eigenvectors of (5) for the trajectories of the fluid elements. These streamlines are termed the entrance and exit streamlines, corresponding to the eigenvectors with the negative and positive eigenvalues, respectively. The angle between the entrance and exit streamlines is related to the flow type, $\alpha$, by:

$$
\tan \left(\frac{1}{2} \theta\right)=\alpha^{\frac{1}{2}} .
$$

This angle could be measured to within \pm 1 degree, yielding an uncertainty of $3 \%$ in $\alpha$. For $\alpha<0$, the flow type could be deduced from the ratio of the length of the minor to major axis of the elliptical streamlines of (4):

$$
\frac{m_{1}}{m_{2}}=(-\alpha)^{\frac{1}{2}}
$$

A series of photographs was taken to determine the relationship between the roller speeds and the flow type $\alpha$. In this series, rollers 1 and 3 were driven at the same rate, and the speeds of rollers 2 and 4 were also identical. The ratio of the speeds of crosswise pairs and the sum of the four rollers speeds (to verify that the flow type was independent of the shear rate for a given ratio) were varied. Figure 6 shows the measured value of $\alpha$ versus the ratio of roller speeds for $-1<\alpha<1$. In later 


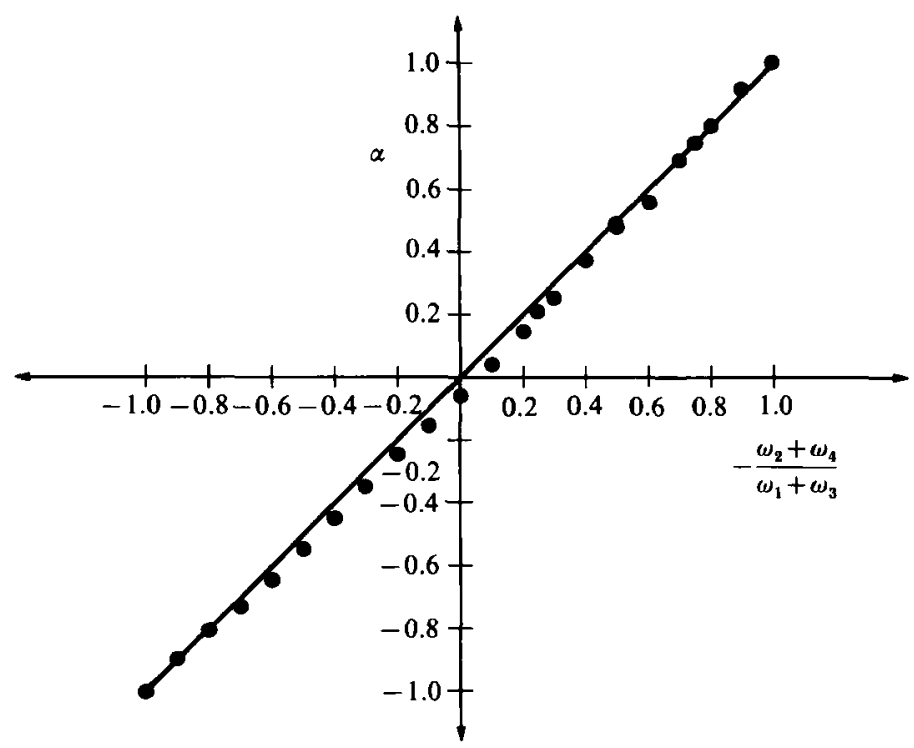

Figure 6. Observed flow type vs. roller speed ratio in four-roll mill.

experiments (discussed below) the constraint that the speeds of diagonal pairs of rollers were equal was removed, and the relationship shown in figure 6 was found to apply in that case as well. Thus figure 6 supplies the function $f_{2}$ in graphical form.

Determination of the relationship between the shear rate and the roller speeds required the more difficult procedure of measuring the position of the beginning and ending of a number of streaklines for each of the time-exposure photographs of the flow. The shear rate could then be calculated from the expression for particle paths $(\alpha>0)$ :

$$
\begin{aligned}
& x=x_{0}\left[\cosh \left(G \alpha^{\frac{1}{2}} t\right)+\frac{1+\alpha}{2 \alpha^{\frac{1}{2}}} \sinh \left(G \alpha^{\frac{1}{2}} t\right)\right]+y_{0}\left[\frac{1-\alpha}{2 \alpha^{\frac{1}{2}}} \sinh \left(G \alpha^{\frac{1}{2}} t\right)\right], \\
& y=y_{0}\left[\cosh \left(G \alpha^{\frac{1}{2}} t\right)-\frac{1+\alpha}{2 \alpha^{\frac{1}{2}}} \sinh \left(G \alpha^{\frac{1}{2}} t\right)\right]-x_{0}\left[\frac{1-\alpha}{2 \alpha^{\frac{1}{2}}} \sinh \left(G \alpha^{\frac{1}{2}} t\right)\right] .
\end{aligned}
$$

Since only flows with positive $\alpha$ were used in this study, the relationship between the shear rate and roller speeds was determined for these flows only. Figure 7 shows the measured shear rate as a function of the speed of rollers 1 and 3 , for $0 \leqslant G \leqslant 10 \mathrm{~s}^{-1}$ with $\alpha$ of $1.0,0.75,0.50$, and 0.25 . As in the determination of the flow types, rollers 1 and 3 were driven at the same rate in these initial studies. The relationship is evidently linear and independent of flow type. The least-squares best fit for the data is given by:

$$
G=0.0399\left(\omega_{1}+\omega_{3}\right) \quad\left(\omega_{1} \text { and } \omega_{3} \text { equal }\right),
$$

where $\omega_{1}$ and $\omega_{3}$ are the speeds of rollers 1 and 3 in revolutions per minute. Later experiments verified that the sum of the speeds of rollers 1 and 3 could indeed be used when the two roller speeds were unequal, provided that the difference between their speeds was less than about $10 \%$. Thus the function $f_{1}(G)$ in (33) is given by:

$$
f_{1}=\frac{G}{0.0399}
$$




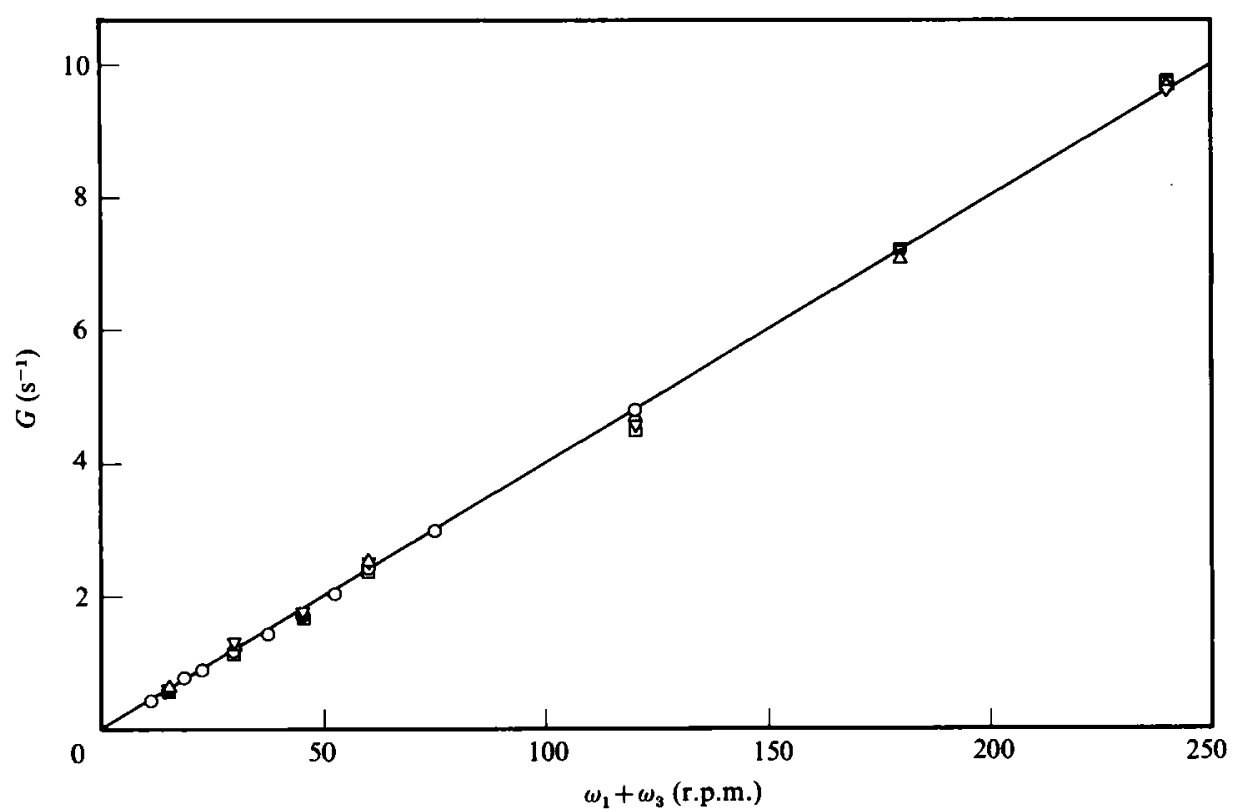

Figure 7. Measured shear rate vs. speed of fastest pair of rollers. $O, \alpha=1 ; \Delta, \alpha=\mathbf{0 . 7 5}$; $\square, \alpha=0.5 ; \nabla, \alpha=0.25$.

To determine the position of the stagnation point as a function of the roller speeds (functions $f_{3}$ and $f_{4}$ in (35) and (36)), photographs similar to those used for determination of the flow type were used, except that a laser beam was shone through the flow field in a fixed position to serve as a reference from which to measure the position of the stagnation point. Initially, attention was restricted to $\alpha=1$, varying the ratio of the speeds of rollers 3 and 4 relative to those of rollers 1 and 2 so that the stagnation point moved along the $x$-axis only. Two different shear rates were studied to verify that the relationship between the stagnation-point position and the ratio of the speeds of the left and right roller pairs was independent of shear rate. The results of these measurements are shown in figure 8 . The stagnation-point position $x_{\mathrm{s}}$ is reduced by the distance $b$ from the centre of the device to the line joining the centres of two adjacent rollers (in the four-roll mill used in these experiments $b=6.35 \mathrm{~cm}$ ). The line in figure 8 represents the empirically determined best fit of the data for $x_{\mathrm{s}}>0$, given by

$$
\frac{x_{\mathrm{s}}}{b}=0.055\left[\frac{\omega_{3}-\omega_{2}}{\omega_{1}-\omega_{4}}-1\right]^{0.825} .
$$

Decomposing the stagnation point into its components $\left(x_{\mathrm{s}}^{\prime}, y_{\mathrm{s}}^{\prime}\right)$ in the rotated coordinate system of figure 5 yields

$$
\begin{aligned}
& \frac{x_{\mathrm{s}}^{\prime}}{b}=0.039\left[\frac{\omega_{3}}{\omega_{1}}-1\right]^{0.825}, \\
& \frac{y_{\mathrm{s}}^{\prime}}{b}=0.039\left[\frac{\omega_{4}}{\omega_{2}}-1\right]^{0.825},
\end{aligned}
$$




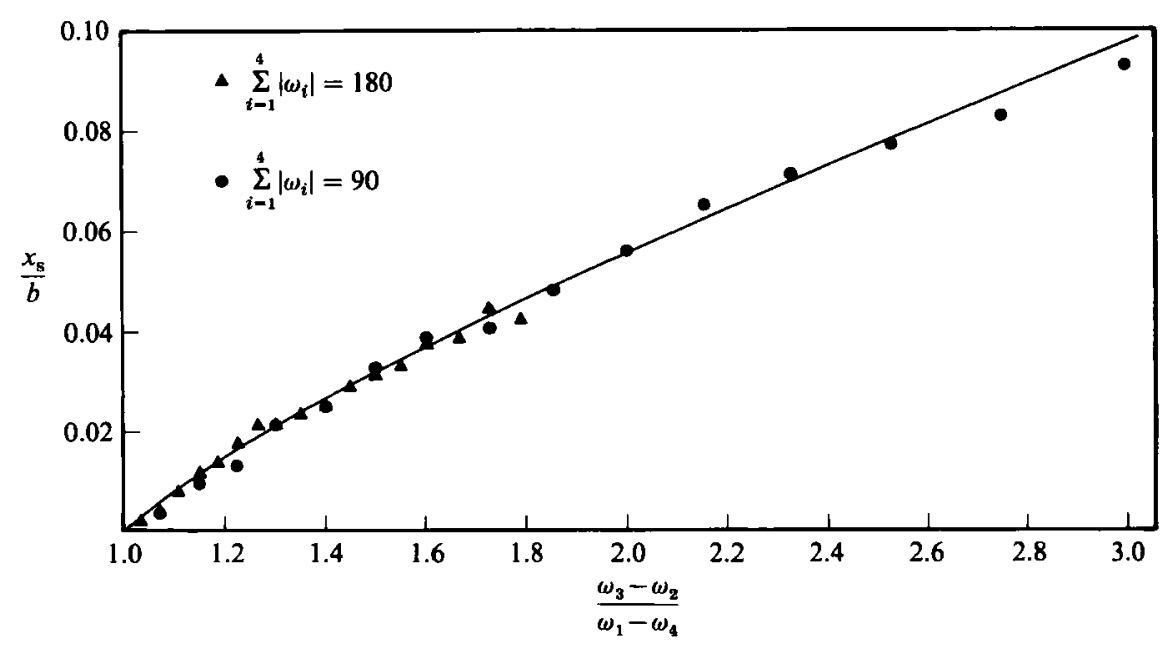

Fiaure 8. Stagnation point position vs. roller speed for $\alpha=1$.

which allows us to determine the functions $f_{3}$ and $f_{4}$ of (35) and (36) above:

$$
\begin{aligned}
& f_{3}\left(x_{\mathrm{s}}^{\prime}\right)=1+51.20\left(\frac{x_{\mathrm{s}}^{\prime}}{b}\right)^{1.2121} \\
& f_{4}\left(y_{\mathrm{s}}^{\prime}\right)=1+51.20\left(\frac{y_{\mathrm{s}}^{\prime}}{b}\right)^{1.2121}
\end{aligned}
$$

These relationships show that for a stagnation-point movement of $0.02 \mathrm{~cm}$ from the device centre (a typical control action), a roller speed change of less than $2 \%$ is required.

A final set of experiments was performed to verify that the generalizations made from the somewhat restrictive conditions used to determine the functions $f_{1}$ to $f_{4}$ were indeed valid when the restrictions were relaxed to allow the four roller speeds to vary independently. Roller speeds required to generate various shear rates and stagnation point positions along the exit streamlines for flows with $\alpha$ of $1.0,0.8,0.6,0.4$, and 0.2 were calculated by determining $f_{1}-f_{4}$ from the calibration results discussed above, and then computing the roller speeds from (38) to (41). The four-roll mill was run with the rollers at these speeds, and photographs similar to those used for the initial calibration were taken. Flow type, shear rate, and stagnation-point position were determined from these photographs and compared to those that the combination of roller speeds was intended to produce. The agreement between the intended and actual flow parameters was generally within about $5 \%$ provided that the stagnationpoint displacement was less than about $0.2 \mathrm{~cm}$. Since the control actions required stagnation-point movements which were generally substantially less than this displacement, the simple model of $\$ 4.2$ was judged adequate for our purposes.

\section{Experimental procedure}

The experiment was run under the supervision of a program written for the DEC computer. This program both controlled the experiment and logged the resulting data. The program was started and the relevant experimental parameters (drop size, 
temperature, flow type, etc.) were entered. The control parameters were also entered. By trial and error, it was determined that $r=0.01$ was satisfactory for all experiments. A value of 0.015 for $\tau$ gave good results for the Pale 4 oil used as a suspending fluid for most of the experiments.

A small drop (about $2 \mathrm{~mm}$ diameter) was introduced into the device. Then, the operator adjusted the value for the light-dark threshold used by the video processor interface. The objective was to select a value so that the intensity at all points inside the drop was below the threshold value, and all points in the background were above that value. The two cameras (video and still cameras) were focused by moving the entire camera-mount system vertically until the still camera came into good focus. This ensured that the video camera was also in focus since the two were set to be focused on the same point at all times. Once these adjustments were made, the computer was directed to centre the drop. The position of the drop was determined from the video data, and the appropriate pair of rollers rotated to centre the drop first in the $x$-direction and then in the $y$-direction. This centring step was necessary to allow the control scheme to start with the drop near the origin.

At this point, a picture of the drop was taken for later determination of its exact size. The camera focus was never changed throughout the experiments, so that the size of the drop could be accurately determined from comparison with a picture of a ruler taken at the same camera settings. Then the experiment was started by another command to the computer. The motors were started at the speeds needed to generate the initial shear rate and flow type, and the control loop entered.

While the experiment was in progress, the operator directed the apparatus through various inputs to the terminal keyboard. Once each time through the control loop (i.e. 5-10 times per second) the program polled the keyboard for commands to expand or contract the active 'window' of the video camera, lower or raise the light-dark threshold value (which was necessary to compensate for the changing light-blocking characteristics of the drop as it deformed), increase or decrease the shear rate, log the current conditions when a still picture was taken, or terminate the experiment.

The sequence of events in a typical run was to start the rollers at a shear rate of about $0.05 \mathrm{~s}^{-1}$. The operator would wait for a time sufficient to allow the drop to come to a steady-state shape. This time depended on the drop viscosity, varying from less than a second for the least viscous fluids up to about a minute for the most viscous drops used. A picture would then be taken with the still camera for later analysis. The shear rate was then increased and the process repeated. A total of about 35 pictures was taken for each viscosity ratio-flow type combination.

As the shear rate was slowly increased, the drop proceeded through a succession of steady shapes of increasing deformation. For most flow type-viscosity ratio combinations, a shear rate was reached where the interfacial tension forces could no longer balance the viscous stresses, and no steady drop shape was possible. This was defined as the point of drop burst for these experiments. Exceptions were for the high-viscosity-ratio drop in the more rotational flows studied.

In cases where drop burst was realized, the flow was continued at the critical shear rate for a variable period after it was clear that no steady shapes existed. The drops became increasingly deformed, but did not break into fragments while the flow was on. At some point, the flow was turned off and the drop motion became governed by interfacial forces. Either the extended drop would fragment into a number of satellite drops through a complicated interfacial tension-driven motion, or it would relax back to the original spherical shape, with the type of behaviour depending on 
the degree of extension prior to turning the flow off and the viscosity ratio. Photographs of deforming and bursting drops for a number of different experiments, and details concerning the results of all experiments can be found in II.

\section{Conclusions}

Our experiments have demonstrated the feasibility of controlling the position of a drop or particle in a four-roll mill based on digitized video data. In general, continuous, real-time control of processes based on video images is not possible, owing to the large information content in even a single video picture. In our case, however, feedback control proved possible with today's technology because both the image processing required (simple thresholding) and the information to be extracted from the image (the centre of mass of an isolated blob) were relatively simple. Control of processes where more information is needed (e.g. location of edges of objects) from the video data requires specialized hardware and software (Wong 1979), and higher computational speeds than generally available in laboratory minicomputers today. However, similar applications in the field of robotics are causing the technology to advance rapidly, to the point where more complex control problems may soon be solved using feedback from video sources.

This work was partially completed while B.J. B. held a National Science Foundation Graduate Fellowship. The research was supported by a grant from the Fluid Mechanics Program of NSF. The authors are grateful for this support.

\section{REFERENCES}

Bentley, B. J. \& Leal, L. G. 1986 An experimental investigation of drop deformation and breakup in steady, two-dimensional linear flows. J. Fluid Mech. 167, 241-283.

Fuller, G. G. \& Leal, L. G. 1981 Flow birefringence of concentrated polymer solutions in two-dimensional flows. J. Polymer. Sci. Polymer. Phys. Ed. 19, 557-587.

Douglas, J. M. 1972 Process Dynamics and Control, Volume 2, Control System Synthesis, pp. 3-20, p. 165. Prentice Hall.

Gieserus, H. 1962 Strömungen mit konstantem Geschwindigkeitsgradienten und die Bewegung von dar in suspendierten Teilchen. Teil II: Ebene Strömungen und eine experimentelle Anordnung zu ihrer Realisierung. Rheol. Acta 2, 113-121.

Grace, H. P. 1971 Dispersion phenomena in high viscosity immiscible fluid systems and application of static mixers as dispersion devices in such systems. Eng. Found. Res. Conf. Mixing, 3rd, Andover, N.H. Republished 1982 in Chem. Engng Commun. 14, 225-277.

Rumscheidt, F. D. \& Mason, S. G. 1961 Particle motions in sheared suspensions. 12. Deformation and burst of fluid drops in shear and hyperbolic flows. J. Colloid Interface Sci. 16, 238-261.

TAYLOR, G. I. 1934 The formation of emulsions in definable fields of flow. Proc. R. Soc. Lond. A 146, 501-523.

Wong, V.S. 1979 Computational structures for extracting edge features from digital images for real-time control applications. Ph.D. dissertation, California Institute of Technology. 